\title{
A new decay mode of higher charmonium
}

\author{
Li-Ye Xiao ${ }^{1,2, a}$, Xin-Zhen Weng ${ }^{1}$, Qi-Fang Lü ${ }^{3,4, b}$, Xian-Hui Zhong ${ }^{3,4, c}$, Shi-Lin Zhu ${ }^{1,2,5, d}$ \\ ${ }^{1}$ School of Physics and State Key Laboratory of Nuclear Physics and Technology, Peking University, Beijing 100871, China \\ ${ }^{2}$ Center of High Energy Physics, Peking University, Beijing 100871, China \\ ${ }^{3}$ Department of Physics, Hunan Normal University, and Key Laboratory of Low-Dimensional Quantum Structures \\ and Quantum Control of Ministry of Education, Changsha 410081, China \\ ${ }^{4}$ Synergetic Innovation Center for Quantum Effects and Applications (SICQEA), Hunan Normal University, Changsha 410081, China \\ ${ }^{5}$ Collaborative Innovation Center of Quantum Matter, Beijing 100871, China
}

Received: 30 May 2018 / Accepted: 18 July 2018 / Published online: 28 July 2018

(C) The Author(s) 2018

\begin{abstract}
We calculate the $\Lambda_{c} \bar{\Lambda}_{c}$ partial decay width of the excited vector charmonium states around $4.6 \mathrm{GeV}$ with the quark pair creation model. We find that the partial decay width of the $\Lambda_{c} \bar{\Lambda}_{c}$ mode can reach up to several MeV for $\psi(4 S, 5 S, 6 S)$. In contrast, the partial $\Lambda_{c} \bar{\Lambda}_{c}$ decay width of the states $\psi(3 D, 4 D, 5 D)$ is less than one $\mathrm{MeV}$. If the enhancement $Y(4630)$ reported by the Belle Collaboration in $\Lambda_{c} \bar{\Lambda}_{c}$ invariant-mass distribution is the same structure as $Y(4660)$, the $Y(4660)$ resonance is most likely to be a $S$-wave charmonium state.
\end{abstract}

\section{Introduction}

Since the Belle Collaboration reported the first member of the family of the charmonium-like states, $X$ (3872), in 2003 [1], a series of charmonium-like states [2], called collectively $X Y Z$ states, have been observed by several major experimental collaborations such as Babar, BESIII, LHCb, CLEO-c and so on. To date, dozens of charmonium-like states [2] have been discovered. The charmonium systems may provide unique clues to the nonperturbative behavior of QCD in the low energy regime and have attracted a great deal of attention from the hadron physics community; see Ref. [3] for a review and references.

The $Y(4660)$ resonance, as the most massive state among the charmonium-like states at present, was first reported by the Belle Collaboration [4] in the process $e^{+} e^{-} \rightarrow$ $\gamma_{\mathrm{ISR}} \pi^{+} \pi^{-} \psi(2 S)$ associated with the $Y(4360)$ resonance in 2007. Its mass and width were determined to be $M=$

\footnotetext{
a e-mail: lyxiao@pku.edu.cn

be-mail: lvqifang@hunnu.edu.cn

ce-mail: zhongxh@hunnu.edu.cn

d e-mail: zhusl@pku.edu.cn
}

$(4664 \pm 16) \mathrm{MeV}$ and $\Gamma=(48 \pm 18) \mathrm{MeV}$, respectively. Later, this state was confirmed by BaBar Collaboration [5] in the $\pi^{+} \pi^{-} \psi(2 S)$ invariant-mass distribution with new data on the $e^{+} e^{-} \rightarrow \gamma_{\mathrm{ISR}} \pi^{+} \pi^{-} \psi(2 S)$ progress with the mass $M=(4669 \pm 24) \mathrm{MeV}$ and width $\Gamma=(104 \pm 58) \mathrm{MeV}$. Since the $Y(4660)$ resonance was produced from the $e^{+} e^{-}$ annihilation, the quantum number is $J^{P C}=1^{--}$. Besides, the Belle Collaboration reported an enhancement, $Y$ (4630), in the cross section of the $e^{+} e^{-} \rightarrow \Lambda_{c}^{+} \Lambda_{c}^{-}$in 2008 [6], whose mass and width are consistent within the errors with those of $Y(4660)$. Hence, these two states may be the same structure although they were observed in different processes [7-9].

Over the past decade, the properties of the charmoniumlike state $Y(4660)$ were extensively explored with various theoretical methods. In the framework of the screened potential model by Li and Chao [10], Y (4660) was a good candidate of the $\psi(6 S)$ state. However, Ding et al. [11] interpreted $Y(4660)$ as the $\psi(5 S)$ state in the flux tube model, which is consistent with the prediction in Refs. [12,13]. Besides the interpretation of the conventional charmonium states, $Y$ (4660) was also interpreted as a tetraquark state [14-23], $f_{0}(980) \psi^{\prime}$ bound state [24-27], baryonium [8,28] and hadrocharmonium state [29] and so on. In addition, van Beveren et al. [30] argued that $Y$ (4660) should not be associated with a resonance pole of the $c \bar{c}$ propagator by analyzing the published Babar data for the reaction $e^{+} e^{-} \rightarrow D^{*} \bar{D}^{*}$ [31]. For the properties of $Y(4630)$, there are many theoretical interpretations as well [32-38].

According to the mass and spin-parity, the possible assignments of $Y(4660)$ as a charmonium state are $\psi(4 S), \psi(5 S)$, $\psi(6 S), \psi(3 D), \psi(4 D)$, or $\psi(5 D)$, which have been listed in Table 1. In the framework of the quark pair creation model (QPC model), we calculate the decay width of the $\Lambda_{c} \bar{\Lambda}_{c}$ mode for those vector charmonium states and obtain that (1) if the $Y(4660)$ is a $S$-wave charmonium state, the par- 
Table 1 The possible assignments of the $Y(4660)$ with the predicted masses $(\mathrm{MeV})$ from various models

\begin{tabular}{llllllll}
\hline State & QM [48] & QM [49] & QM [50] & SSE/EA [51] & NR/GI [52] & SP [10] & LP/SP [53] \\
\hline$\psi\left(4^{3} S_{1}\right)$ & 4625 & 4450 & 4389 & $4398 / 4426$ & $4406 / 4450$ & 4273 & $4412 / 4281$ \\
$\psi\left(5^{3} S_{1}\right)$ & $\ldots$ & $\ldots$ & 4641 & $4642 / 4672$ & $\ldots$ & 4463 & $4711 / 4472$ \\
$\psi\left(6^{3} S_{1}\right)$ & $\ldots$ & $\ldots$ & $\ldots$ & $4804 / 4828$ & $\ldots$ & 4608 & $\ldots$ \\
$\psi\left(3^{3} D_{1}\right)$ & $\ldots$ & 4520 & 4426 & $4464 / 4477$ & $\ldots$ & 4317 & $4478 / 4336$ \\
$\psi\left(4^{3} D_{1}\right)$ & $\ldots$ & $\ldots$ & 4641 & $4690 / 4707$ & $\ldots$ & $\ldots$ & $\ldots$ \\
$\psi\left(5^{3} D_{1}\right)$ & $\ldots$ & $\ldots$ & $\ldots$ & $4840 / 4855$ & $\ldots$ & $\ldots$ & $\ldots$ \\
\hline
\end{tabular}

tial decay width of the $\Lambda_{c} \bar{\Lambda}_{c}$ mode can reach several MeV. However, if the $Y(4660)$ is a $D$-wave charmonium state, the partial decay width of the $\Lambda_{c} \bar{\Lambda}_{c}$ mode should be less than one $\mathrm{MeV}$. (2) If the enhancement $Y$ (4630) reported by Belle Collaboration in $\Lambda_{c} \bar{\Lambda}_{c}$ invariant-mass distribution is the same structure as $Y(4660)$, the $Y(4660)$ resonance is most likely to be a $S$-wave charmonium state.

This paper is organized as follows. In Sect. 2 we give a brief introduction of the QPC model. Then, we present our numerical results and discussions in Sect. 3 and summarize our results in Sect. 4.

\section{An introduction of the ${ }^{3} P_{0}$ model}

The QPC model is known as ${ }^{3} P_{0}$ model, which was first proposed by Micu [39], Carlitz and Kislinger [40], and further developed by the Orsay group [41-44]. This model is widely used to study the OZI-allowed strong decays of hadrons. In the model, a quark pair $q \bar{q}$ is created from the vacuum and then regroups with the quarks within the initial hadron to produce two outgoing hadrons. In particular, the interaction Hamiltonian for one quark pair creation was assumed as [4547]

$H_{q \bar{q}}=\gamma \sum_{f} 2 m_{f} \int d^{3} x \bar{\psi}_{f} \psi_{f}$,

where $m_{f}$ is the constituent quark mass of flavor $f, \psi_{f}$ denotes a Dirac quark field, and $\gamma$ is a dimensionless parameter describing the $q \bar{q}$ pair-production strength, which is usually fixed by fitting to the well measured decay widths.

In this work, we extend the ${ }^{3} P_{0}$ model to study the charmonium system decaying into a $\Lambda_{c} \bar{\Lambda}_{c}$ pair. For this type of reaction, it is necessary to create two light quark pairs, which is shown in Fig. 1. In the framework of the ${ }^{3} P_{0}$

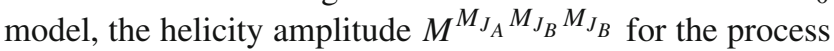
of $Y(4660)(A) \rightarrow \Lambda_{C}(B)+\bar{\Lambda}_{c}(C)$ reads

$$
\begin{gathered}
\delta^{3}\left(\mathbf{p}_{A}-\mathbf{p}_{B}-\mathbf{p}_{C}\right) M^{M_{J_{A}} M_{J_{B}} M_{J_{B}}} \\
=\frac{\sum_{k}\left\langle B C\left|H_{q \bar{q}}\right| k\right\rangle\left\langle k\left|H_{q \bar{q}}\right| A\right\rangle}{E_{k}-E_{A}} .
\end{gathered}
$$

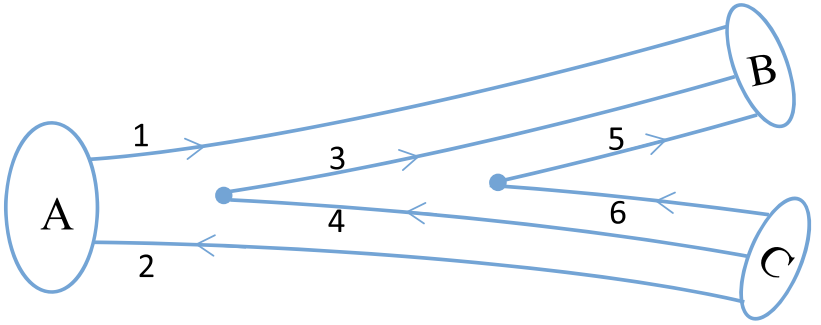

Fig. 1 The charmonium system decays into a $\Lambda_{c} \bar{\Lambda}_{c}$ pair

Here, $\mathbf{p}_{A}\left(\mathbf{p}_{B} / \mathbf{p}_{C}\right)$ represents the momentum of the hadron $A(B / C) . E_{k}$ and $E_{A}$ stand for the energy of the intermediate state $k$ and initial state $A$, respectively. To simplify the calculations, we take $E_{k}-E_{A}$ as a constant, namely $E_{k}-E_{A} \equiv 2 m_{q}$. This approximation is reasonable because the intermediate state just have two more created quarks compared to the initial state at the quark level. On the other hand, the allowed intermediate states with the spin parity $J^{P C}=$ $1^{--}$are $D \bar{D}_{1}, D^{*} \bar{D}_{1}, D^{*} \bar{D}_{0}, D^{*} \bar{D}_{2}, J / \Psi f_{0}(500) / f_{0}(600)$, $h_{c}(1 P) \eta, h_{c}(1 P) \pi, \chi_{c 0}(1 P) \omega(782)$ and $\chi_{c 2}(1 P) \omega(782)$ and tetraquark states [14] and so on. Their masses are about $\sim 4.0 \mathrm{GeV}$. Thus, we use $E_{k}-E_{A} \sim 0.6 \mathrm{GeV} \sim 2 m_{q}$. Under the above approximation, we can rewrite the Eq. (2) as

$\delta^{3}\left(\mathbf{p}_{A}-\mathbf{p}_{B}-\mathbf{p}_{C}\right) M^{M_{J_{A}} M_{J_{B}} M_{J_{B}}}=\frac{\left\langle B C\left|H_{q \bar{q}} H_{q \bar{q}}\right| A\right\rangle}{2 m_{q}}$,

where $m_{q}$ is the mass of the created quark pair.

In the nonrelativistic limit, the transition operator for the two quark pairs creation under the ${ }^{3} P_{0}$ model is given by

$$
\begin{aligned}
T= & \frac{9 \gamma^{2}}{4 m_{\mu}} \sum_{m, m^{\prime}}\langle 1 m ; 1-m \mid 00\rangle\left\langle 1 m^{\prime} ; 1-m^{\prime} \mid 00\right\rangle \\
& \times \int d^{3} \mathbf{p}_{3} d^{3} \mathbf{p}_{4} d^{3} \mathbf{p}_{5} d^{3} \mathbf{p}_{6} \delta^{3}\left(\mathbf{p}_{3}+\mathbf{p}_{4}\right) \delta^{3}\left(\mathbf{p}_{5}+\mathbf{p}_{6}\right) \\
& \times \varphi_{0}^{34} \omega_{0}^{34} \chi_{1,-m}^{34} \mathcal{Y}_{1}^{m}\left(\frac{\mathbf{p}_{3}-\mathbf{p}_{4}}{2}\right) a_{3 i}^{\dagger} b_{4 j}^{\dagger} \\
& \times \varphi_{0}^{56} \omega_{0}^{56} \chi_{1,-m^{\prime}}^{56} \mathcal{Y}_{1}^{m^{\prime}}\left(\frac{\mathbf{p}_{5}-\mathbf{p}_{6}}{2}\right) a_{5 i}^{\dagger} b_{6 j}^{\dagger},
\end{aligned}
$$


where $\mathbf{p}_{i}(i=3,4,5,6)$ corresponds to the three-vector momentum of the $i$ th quark within the two created quark pairs. $\varphi_{0}=(u \bar{u}+d \bar{d}+s \bar{s}) / \sqrt{3}$ and $\omega_{0}=\delta_{i j}$ correspond to the flavor function and color singlet, respectively. The solid harmonic polynomial $\mathcal{Y}_{1}^{m\left(m^{\prime}\right)}(\mathbf{p}) \equiv|\mathbf{p}| Y_{1}^{m\left(m^{\prime}\right)}\left(\theta_{p}, \phi_{p}\right)$ stand for the $P$-wave quark pairs, and $\chi_{1,-m\left(m^{\prime}\right)}$ are the spin triplet states for the created quark pairs. $a_{i}^{\dagger} b_{j}^{\dagger}$ is the creation operator denoting the quark pairs creation in the vacuum.

Adopting the definition of the mock state [54], the meson $(A)$ and baryon $(B)$ states are defined as, respectively,

$$
\begin{aligned}
& \left|A\left(N_{A}{ }^{2 S_{A}+1} L_{A} J_{A} M_{J_{A}}\right)\left(\mathbf{p}_{A}\right)\right\rangle=\sqrt{2 E_{A}} \varphi_{A}^{12} \omega_{A}^{12} \\
& \times \sum_{M_{L_{A}}, M_{S_{A}}}\left\langle L_{A} M_{L_{A}} ; S_{A} M_{S_{A}} \mid J_{A} M_{J_{A}}\right\rangle \\
& \times \int d^{3} \mathbf{p}_{1} d^{3} \mathbf{p}_{2} \delta^{3}\left(\mathbf{p}_{1}+\mathbf{p}_{2}-\mathbf{p}_{A}\right) \\
& \times \Psi_{N_{A} L_{A} M_{L_{A}}\left(\mathbf{p}_{1}, \mathbf{p}_{2}\right)} \chi_{S_{A} M_{S_{A}}}^{12}\left|q_{1}\left(\mathbf{p}_{1}\right) q_{2}\left(\mathbf{p}_{2}\right)\right\rangle, \\
& \left|B\left(N_{B}{ }^{2 S_{B}+1} L_{B} J_{B} M_{J_{B}}\right)\left(\mathbf{p}_{B}\right)\right\rangle=\sqrt{2 E_{B}} \varphi_{B}^{135} \omega_{B}^{135} \\
& \times \sum_{M_{L_{B}}, M_{S_{B}}}\left\langle L_{B} M_{L_{B}} ; S_{B} M_{S_{B}} \mid J_{B} M_{J_{B}}\right\rangle \\
& \times \int d^{3} \mathbf{p}_{1} d^{3} \mathbf{p}_{3} d^{3} \mathbf{p}_{5} \delta^{3}\left(\mathbf{p}_{1}+\mathbf{p}_{3}+\mathbf{p}_{5}-\mathbf{p}_{B}\right) \\
& \times \Psi_{N_{B} L_{B} M_{L_{B}}\left(\mathbf{p}_{1}, \mathbf{p}_{3}, \mathbf{p}_{5}\right)} \chi_{S_{B} M_{S_{B}}}^{135}\left|q_{1}\left(\mathbf{p}_{1}\right) q_{3}\left(\mathbf{p}_{3}\right) q_{5}\left(\mathbf{p}_{5}\right)\right\rangle .
\end{aligned}
$$

The $\mathbf{p}_{i}(i=1,2,3,5)$ denotes the momentum of quarks in hadrons $A$ and $B$. Since the ${ }^{3} P_{0}$ model obtains a reasonable description of the decay properties of many mesons with the simple harmonic oscillator (SHO) wave functions, which are a de facto standard for many nonrelativistic quark model calculations [45,46,55-57], we adopt the SHO wave functions to describe the space-wave functions of the baryons in this work. Meanwhile, with the simple SHO wave functions the decay amplitudes can be calculated analytically, which are helpful to understand the general decay behavior. The SHO wave function of a meson without radial excitations reads

$$
\begin{aligned}
\psi_{l m}^{0}(\mathbf{p})= & (-i)^{l}\left[\frac{2^{l+2}}{\sqrt{\pi}(2 l+1) ! !}\right]^{\frac{1}{2}}\left(\frac{1}{\beta}\right)^{l+\frac{3}{2}} \\
& \times \exp \left(-\frac{\mathbf{p}_{R}^{2}}{2 \beta^{2}}\right) \mathcal{Y}_{l}^{m}(\mathbf{p})
\end{aligned}
$$

and the ground state space-wave function of a baryon reads

$\psi_{0,0}=3^{\frac{3}{4}}\left(\frac{1}{\pi \alpha_{\rho}^{2}}\right)^{\frac{3}{4}}\left(\frac{1}{\pi \alpha_{\lambda}^{2}}\right)^{\frac{3}{4}} \exp \left(-\frac{\mathbf{p}_{\rho}^{2}}{2 \alpha_{\rho}^{2}}-\frac{\mathbf{p}_{\lambda}^{2}}{2 \alpha_{\lambda}^{2}}\right)$.

Here the $\mathbf{p}_{R}$ stands for the relative momentum between the quark and antiquark within the meson. $\mathbf{p}_{\rho}$ and $\mathbf{p}_{\lambda}$ stand for the momentum corresponding to $\rho$ and $\lambda$ jacobi coordinates (see Fig. 2), respectively. Thus, we can obtain the helicity amplitude in the center of mass frame,

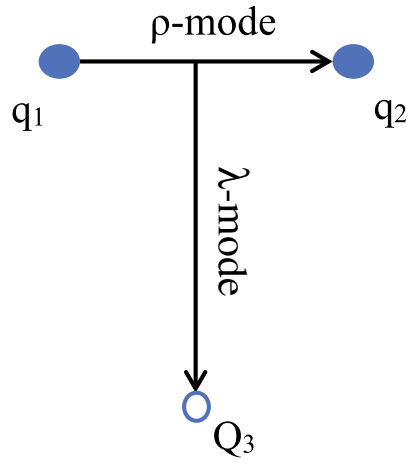

Fig. 2 The $\rho$ - and $\lambda$-mode excitations of the $c q q$ system where $\rho$ and $\lambda$ are the jacobi coordinates defined as $\rho=\frac{\mathbf{r}_{1}-\mathbf{r}_{2}}{\sqrt{2}}$ and $\lambda=\frac{\mathbf{r}_{1}+\mathbf{r}_{2}-2 \mathbf{r}_{3}}{\sqrt{6}}$, respectively. $q_{1}$ and $q_{2}$ represent the light $(u, d)$ quark, and $Q_{3}$ represents the charm quark

$$
\begin{aligned}
& \mathcal{M}^{M_{J_{A}} M_{J_{B}} M_{J_{C}}}(A \rightarrow B+C)=\frac{9 \gamma^{2}}{4 m_{\mu}} \sqrt{8 E_{A} E_{B} E_{C}} \\
& \times \prod_{A, B, C}\left\langle\chi_{S_{B} M_{S_{B}}}^{135} \chi_{S_{C} M_{S_{C}}^{246}} \mid \chi_{S_{A} M_{S_{A}}^{12}}^{34} \chi_{1-m}^{34} \chi_{1-m^{\prime}}^{56}\right\rangle \\
& \quad \times\left\langle\omega_{B}^{135} \omega_{C}^{246} \mid \omega_{A}^{12} \omega_{0}^{34} \omega_{0}^{56}\right\rangle\left\langle\varphi_{B}^{135} \varphi_{C}^{246} \mid \varphi_{A}^{12} \varphi_{0}^{34} \varphi_{0}^{56}\right\rangle I_{M_{L_{B}}, M_{L_{C}}}^{M_{L_{A}}, m, m^{\prime}}(\mathbf{p}) .
\end{aligned}
$$

Here, $I_{M_{L_{B}}, M_{L_{C}}}^{M_{L_{A}}, m, m^{\prime}}(\mathbf{p})$ stands the spatial integral and more detailed calculations are shown in the Appendix. The $\prod_{A, B, C}$ corresponds to the Clebsch-Gorden coefficients for the two created quark pairs, initial and final hadrons, which come from the couplings among the spin, orbital, and total angular momentum. Its specific expression is

$$
\begin{aligned}
& \sum\left\langle L_{A} M_{L_{A}} ; S_{A} M_{S_{A}} \mid J_{A} M_{J_{A}}\right\rangle\langle 1 m ; 1-m \mid 00\rangle\left\langle 1 m^{\prime} ; 1-m^{\prime} \mid 00\right\rangle \\
& \quad \times\left\langle L_{B} M_{L_{B}} ; S_{B} M_{S_{B}} \mid J_{B} M_{J_{B}}\right\rangle\left\langle L_{C} M_{L_{C}} ; S_{C} M_{S_{C}} \mid J_{C} M_{J_{C}}\right\rangle .
\end{aligned}
$$

Finally, with the relativistic phase space, the hadronic decay width $\Gamma[A \rightarrow B C]$ reads

$$
\begin{aligned}
\Gamma[A \rightarrow B C]= & \pi^{2} \frac{|\mathbf{p}|}{M_{A}^{2}} \frac{1}{2 J_{A}+1} \\
& \times \sum_{M_{J_{A}}, M_{J_{B}}, M_{J_{C}}} \mid \mathcal{M}^{\left.M_{J_{A}} M_{J_{B}} M_{J_{C}}\right|^{2} .}
\end{aligned}
$$

In the equation, the momentum $\mathbf{p}$ of the daughter baryon in the center of mass frame of the parent baryon $A$ is

$$
|\mathbf{p}|=\frac{\sqrt{\left[M_{A}^{2}-\left(M_{B}-M_{C}\right)^{2}\right]\left[M_{A}^{2}-\left(M_{B}+M_{C}\right)^{2}\right]}}{2 M_{A}} .
$$

In the present calculation, we adopt $m_{u}=m_{d}=330 \mathrm{MeV}$ and $m_{c}=1628 \mathrm{MeV}$ for the constituent quark masses. The masses of the baryons $\Lambda_{c}$ and $\bar{\Lambda}_{c}$ are taken as $m_{\Lambda_{c}}=m_{\bar{\Lambda}_{c}}=2286.46 \mathrm{MeV}$, which are from the Particle Data Group [2]. The harmonic oscillator strength $\beta$ for the excitation between the two charm quarks in initial charmonium 
system (see Fig. 2) is adopted as $500 \mathrm{MeV}$ [52]. The parameter $\alpha_{\rho}$ of the $\rho$-mode excitation between the two light quarks in final single-heavy baryons is taken the average value as $\alpha_{\rho}=400 \mathrm{MeV}$, and the other harmonic oscillator parameter $\alpha_{\lambda}$ is obtained with the following relation [58-60]:

$\alpha_{\lambda}=\left(\frac{3 m_{Q}}{2 m_{q}+m_{Q}}\right)^{1 / 4} \alpha_{\rho}$.

In this equation, $m_{Q}$ stands for the constituent quark mass of charmed quark, and $m_{q}$ responds the constituent quark mass of light quark $(q=u, d)$.

For the strength of the quark pair creation from the vacuum, we adopt the definition from Ref. [61], where $\gamma$ is a scale-dependent form,

$\gamma(\mu)=\frac{\gamma_{0}}{\log \left(\frac{\mu}{\mu_{0}}\right)}$.

Here, $\mu$ is the reduced mass of the quark-antiquark in the decaying meson, and $\gamma_{0}=0.81 \pm 0.02$ and $\mu_{0}=(49.84 \pm$ 2.58) $\mathrm{MeV}$. According to Eq. (14), we get $\gamma(\mu)=0.29$ with the mass of $m_{c}=1628 \mathrm{MeV}$. So, the strength of the quark pair creation employed in this work is $\gamma=5.04$ which is $\sqrt{96 \pi}$ times of that in Ref. [61] due to a different definition $[46,52,62]$. The uncertainty of the strength $\gamma$ is around $30 \%[47,62-64]$ and the partial decay width is proportional to $\gamma^{4}$, so the uncertainty of our theoretical results may be quite large.

\section{Calculations and results}

The quantum number of the $Y$ (4660) resonance is determined to be $J^{P}=1^{--}$from the $e^{+} e^{-}$annihilation. The average values of mass and width listed in PDG [2] are $M=$ (4643 \pm 9) $\mathrm{MeV}$ and $\Gamma_{\text {total }}=72 \pm 11 \mathrm{MeV}$, respectively. Around $4660 \mathrm{MeV}$, there are six vector charmonium states, which are $\psi(4 S), \psi(5 S), \psi(6 S), \psi(3 D), \psi(4 D)$, and $\psi(5 D)$. In the following, we will discuss the decay properties of these states.

\section{$3.1 S$ wave}

The theoretical mass of $\psi\left(5^{3} S_{1}\right)$ is about $4.64 \mathrm{GeV}$ (see Table 1), which is in good agreement with the mass of $Y$ (4660) in PDG [2]. In addition, via evaluating the open flavor strong decays, some people interpreted $Y(4660)$ as a $\psi(5 S)$ state in the flux tube model [11] and QPC model [12], respectively. As the possibel assignment of $Y(4660)$, it is crucial to study the decay properties of the $\psi\left(5^{3} S_{1}\right)$.

According to our calculations, we get

$\Gamma\left[\psi\left(5^{3} S_{1}\right) \rightarrow \Lambda_{c} \bar{\Lambda}_{c}\right] \sim 2.44 \mathrm{MeV}$
Table 2 The $\Lambda_{c} \bar{\Lambda}_{c}$ partial decay widths (MeV) of the vector charmonium with a mass of $M=4643 \mathrm{MeV}$

\begin{tabular}{llll}
\hline State & $\psi\left(4^{3} S_{1}\right)$ & $\psi\left(5^{3} S_{1}\right)$ & $\psi\left(6^{3} S_{1}\right)$ \\
$\Gamma_{\Lambda_{c} \bar{\Lambda}_{c}}$ & 6.57 & 2.44 & 0.84 \\
State & $\psi\left(3^{3} D_{1}\right)$ & $\psi\left(4^{3} D_{1}\right)$ & $\psi\left(5^{3} D_{1}\right)$ \\
$\Gamma_{\Lambda_{c} \bar{\Lambda}_{c}}$ & 0.33 & 0.19 & 0.09 \\
\hline
\end{tabular}

with a mass of $M=4643 \mathrm{MeV}$ (see Table 2). Combing the measured width of $Y$ (4660), we further predict the branching ratio

$\mathcal{B}\left[\psi\left(5^{3} S_{1}\right) \rightarrow \Lambda_{c} \bar{\Lambda}_{c}\right] \sim 3 \%$.

The sizeable branching ratio indicates that this state has a good potential to be observed in the $\Lambda_{c} \bar{\Lambda}_{c}$ decay channel.

Meanwhile, considering the uncertainties of the predicted mass, we plot the variation of the partial decay width of the $\Lambda_{c} \bar{\Lambda}_{c}$ mode as a function of the mass of the state $\psi\left(5^{3} S_{1}\right)$ in Fig. 3. The decay width of the $\Lambda_{c} \bar{\Lambda}_{c}$ mode increases with the mass increasing in the range of (4580-4602) MeV, and the width can reach up to $\Gamma \sim 3.4 \mathrm{MeV}$. However, when the mass increases in the range of (4603-4800) MeV, the partial decay width decreases.

Besides $\psi\left(5^{3} S_{1}\right)$, we also investigate the decay properties of the states $\psi\left(4^{3} S_{1}\right)$ and $\psi\left(6^{3} S_{1}\right)$. The predicted masses of these two states are listed in Table 1. From the Table 1 it is seen that the possibility of $Y(4660)$ taken as the state $\psi\left(4^{3} S_{1}\right)$ or $\psi\left(6^{3} S_{1}\right)$ can't be excluded completely. Fixing the masses at $M=4643 \mathrm{MeV}$, we obtain

$\Gamma\left[\psi\left(4^{3} S_{1}\right) \rightarrow \Lambda_{c} \bar{\Lambda}_{c}\right] \sim 6.57 \mathrm{MeV}$,

and

$\Gamma\left[\psi\left(6^{3} S_{1}\right) \rightarrow \Lambda_{c} \bar{\Lambda}_{c}\right] \sim 0.84 \mathrm{MeV}$.

The predicted decay widths are large enough to be observed in experiments. Moreover,

$\frac{\Gamma\left[\psi\left(4^{3} S_{1}\right) \rightarrow \Lambda_{c} \bar{\Lambda}_{c}\right]}{\Gamma\left[\psi\left(5^{3} S_{1}\right) \rightarrow \Lambda_{c} \bar{\Lambda}_{c}\right]} \sim 2.7$.

The branching ratio of $\psi\left(4^{3} S_{1}\right)$ decaying into $\Lambda_{c} \bar{\Lambda}_{c}$ pair is larger than that of $\psi\left(5^{3} S_{1}\right)$.

Meanwhile, if the $Y(4660)$ resonance corresponds to $\psi\left(6^{3} S_{1}\right)$, the branching ratio is predicted to be

$\mathcal{B}\left[\psi\left(6^{3} S_{1}\right) \rightarrow \Lambda_{c} \bar{\Lambda}_{c}\right] \sim 1 \%$.

This branching ratio is the smallest, while it is quite large compared to the ratio $\left(\mathcal{O}\left(10^{-3}\right) \sim \mathcal{O}\left(10^{-5}\right)\right)$ of other charmonium states decaying into the baryon-antibaryon pair [2].

In addition, we also plot the decay width of the $\psi\left(4^{3} S_{1}\right)$ and $\psi\left(6^{3} S_{1}\right)$ as a function of the mass in the range of $M=$ (4580-4800) MeV in Fig. 3. The variation curves between 

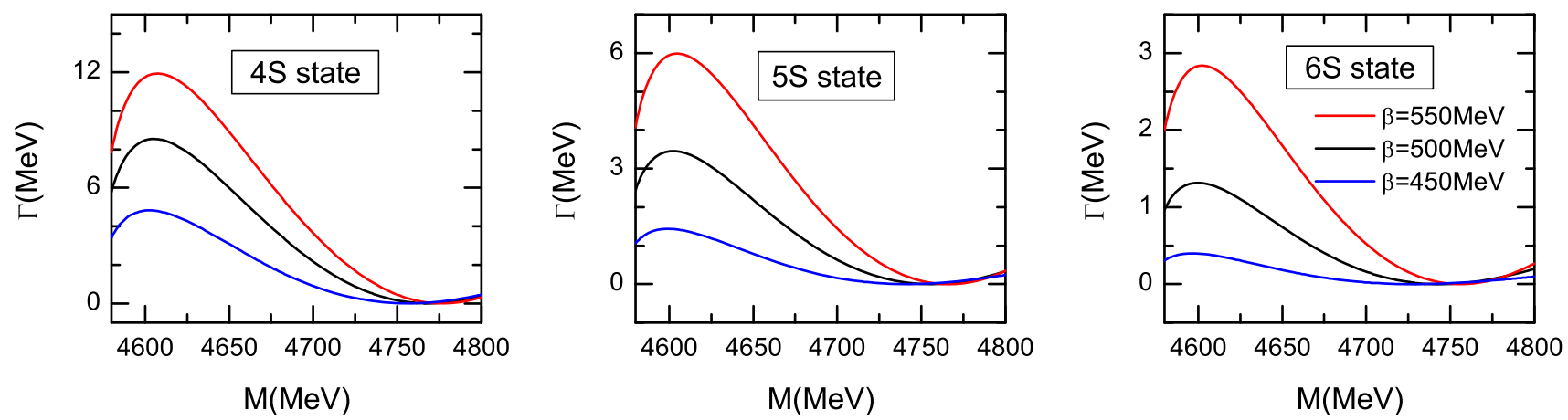

Fig. 3 The variation of the $\Lambda_{c} \bar{\Lambda}_{c}$ decay width with the mass of the S-wave vector charmonium. The blue, black, and red lines correspond to the predictions with different values of the harmonic oscillator strength $\beta=450,500$, and $550 \mathrm{MeV}$, respectively
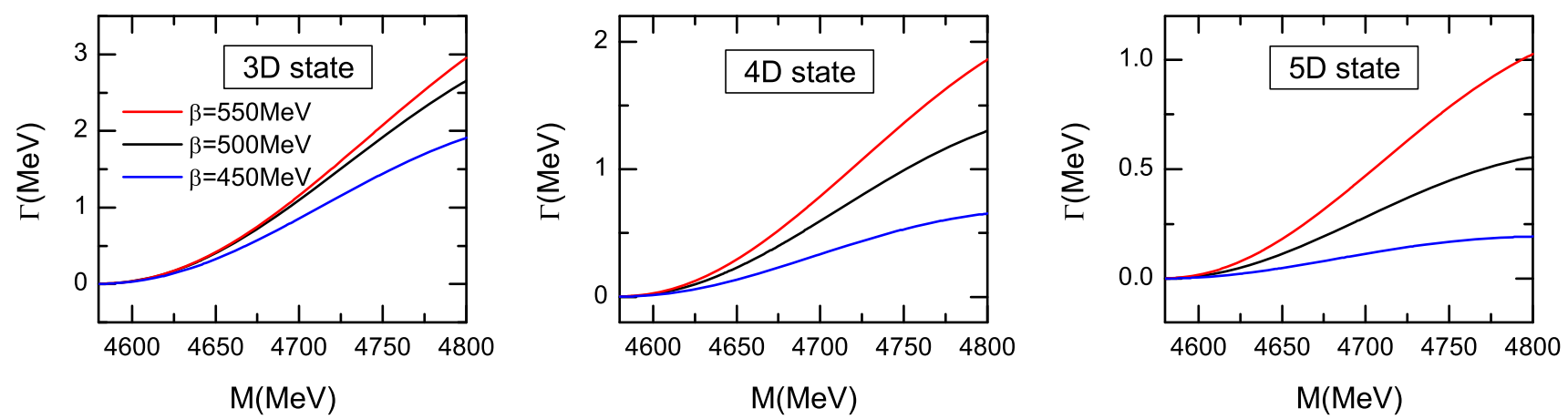

Fig. 4 The variation of the $\Lambda_{c} \bar{\Lambda}_{c}$ decay width with the mass of the D-wave vector charmonium. The blue, black, and red lines correspond to the predictions with different values of the harmonic oscillator strength $\beta=450,500$, and $550 \mathrm{MeV}$, respectively

the partial decay width and the mass for these two states are similar to that for $\psi\left(5^{3} S_{1}\right)$.

In brief, we have calculated the $\Lambda_{c} \bar{\Lambda}_{c}$ partial decay widths of the three $S$-wave states $\psi\left(4^{3} S_{1}\right), \psi\left(5^{3} S_{1}\right)$, and $\psi\left(6^{3} S_{1}\right)$ with the QPC model. According to our predictions, the $\Lambda_{c} \bar{\Lambda}_{c}$ decay width can reach up to a few MeV. If $Y(4660)$ is a vector charmonium, it is very likely to be found in the $\Lambda_{c} \bar{\Lambda}_{c}$ channel.

\section{$3.2 D$ wave}

The predicted mass of the state $\psi\left(4^{3} D_{1}\right)$ is listed in Table 1 . This state is a good candidate of the $Y(4660)$ resonance. So it is necessary to investigate the decay properties of $\psi\left(4^{3} D_{1}\right)$.

In the same way, we fix the mass of $\psi\left(4^{3} D_{1}\right)$ at $M=4643$ $\mathrm{MeV}$ firstly. Then, we obtain the partial decay width

$\Gamma\left[\psi\left(4^{3} D_{1}\right) \rightarrow \Lambda_{c} \bar{\Lambda}_{c}\right] \sim 0.19 \mathrm{MeV}$.

This width seems not large, but it is enough to be observed in this decay channel in experiments. Moreover, the branching ratio is predicted to be

$\mathcal{B}\left[\psi\left(4^{3} D_{1}\right) \rightarrow \Lambda_{c} \bar{\Lambda}_{c}\right] \sim 0.3 \%$.

We also plot the variation of the $\Lambda_{c} \bar{\Lambda}_{c}$ decay width as a function of the mass in Fig. 4. From the Fig. 4, the partial decay width for $\psi\left(4^{3} D_{1}\right)$ decaying into the $\Lambda_{c} \bar{\Lambda}_{c}$ pair is less than $\sim 1.3 \mathrm{MeV}$ in the range of $(4580-4800) \mathrm{MeV}$.

Furthermore, we analyze the decay properties of the states $\psi\left(3^{3} D_{1}\right)$ and $\psi\left(5^{3} D_{1}\right)$, and collect their predicted masses in Table 1. From the Table 1, the theoretical masses are either about (100 200) MeV lighter or heavier than the mass of $Y$ (4660) in PDG [2]. We also study the decay properties of the two states in this work.

Taking the masses of the $\psi\left(3^{3} D_{1}\right)$ and $\psi\left(5^{3} D_{1}\right)$ as $M=$ $4643 \mathrm{MeV}$, we get that the partial decay widths are

$\Gamma\left[\psi\left(3^{3} D_{1}\right) \rightarrow \Lambda_{c} \bar{\Lambda}_{c}\right] \sim 0.33 \mathrm{MeV}$

and

$\Gamma\left[\psi\left(5^{3} D_{1}\right) \rightarrow \Lambda_{c} \bar{\Lambda}_{c}\right] \sim 0.09 \mathrm{MeV}$.

If the $Y(4660)$ were the state $\psi\left(5^{3} D_{1}\right)$, one would be very hard to observe $Y$ (4660) in the $\Lambda_{c} \bar{\Lambda}_{c}$ channel.

The predicted masses of the states $\psi\left(3^{3} D_{1}\right)$ and $\psi\left(5^{3} D_{1}\right)$ certainly have a large uncertainty, which may bring uncertainties to our theoretical results. To investigate this effect, we plot the partial decay widths of these two states as functions of the masses in Fig. 4 as well.

The $\Lambda_{c} \bar{\Lambda}_{c}$ decay widths of the $D$-wave states are less than one $\mathrm{MeV}$. The $\Lambda_{c} \bar{\Lambda}_{c}$ decay width ratio between the $S$-wave states and $D$-wave states is $\mathcal{O}(10)$. If $Y(4660)$ turns out to 
be a $S$-wave state, it has a good potential to be observed in the $\Lambda_{c} \bar{\Lambda}_{c}$ channel.

\subsection{The effect of $\beta$}

We have considered six excited vector charmonium states around $4.6 \mathrm{Gev}$ and investigated their $\Lambda_{c} \bar{\Lambda}_{c}$ partial decay width. In the present work, all of the theoretical predictions are obtained with the parameter $\beta=500 \mathrm{MeV}$. However, the harmonic oscillator parameter $\beta$ for the excitation between the charm quarks in initial charmonium system is not determined precisely, which bares a large uncertainty. To investigate the uncertainties of the parameter $\beta$, we further consider the decay properties as a function of the mass with two different $\beta$ values: $\beta=450,550 \mathrm{MeV}$. The numerical results are shown in Figs. 3 and 4. One notes that the bigger $\beta$ value leads to a larger decay width. Our main predictions hold in a reasonable range of the parameter $\beta$.

\section{Summary}

In the present work, we calculate the $\Lambda_{c} \bar{\Lambda}_{c}$ partial decay width of the excited vector charmonium around $4.6 \mathrm{GeV}$, including $\psi(4 S, 5 S, 6 S)$ and $\psi(3 D, 4 D, 5 D)$. The $\Lambda_{c} \bar{\Lambda}_{c}$ mode is not kinematically allowed for the charmonium states below $4.6 \mathrm{GeV}$. This OZI allowed mode provides a new tool to explore the higher charmonium, which will be produced abundantly at Belle-II. We extend the original ${ }^{3} P_{0}$ model and consider the creation of two light $q \bar{q}$ pairs from the vacuum, which is the first attempt along this direction in literatures up to our knowledge.

Based on our calculations, the decay widths of the $S$-wave states decaying into the $\Lambda_{c} \bar{\Lambda}_{c}$ pair are about a few $\mathrm{MeV}$, while the $\Lambda_{c} \bar{\Lambda}_{c}$ decay widths of the $D$-wave states are less than one $\mathrm{MeV}$. The $\Lambda_{c} \bar{\Lambda}_{c}$ decay width ratio between the $S$ wave states and the $D$-wave states is $\mathcal{O}(10)$. If the $Y(4660)$ is one of the $S$-wave states considered in this work, it may be observed in the $\Lambda_{c} \bar{\Lambda}_{c}$ channel. Moreover, if the enhancement $Y(4630)$ reported by Belle collaboration in $\Lambda_{c} \bar{\Lambda}_{c}$ invariantmass distribution is the same structure as the $Y(4660)$, the $Y(4660)$ is very likely to be a $S$-wave charmonium state. On the other hand, it will be very difficult to observe the excited D-wave vector charmonium in the $\Lambda_{c} \bar{\Lambda}_{c}$ channel. In other words, the $\Lambda_{c} \bar{\Lambda}_{c}$ mode can be used to pin down the internal structure of the vector charmonium.

Acknowledgements We would like to thank Xiao-Lin Chen and WeiZhen Deng for very helpful discussions. This work is supported by the National Natural Science Foundation of China under Grants no. $11621131001,11575008,11775078$ and 11705056 . This work is also in part supported by China Postdoctoral Science Foundation under Grant no. 2017M620492 and by National Key Basic Research Program of China (2015CB856700).
Open Access This article is distributed under the terms of the Creative Commons Attribution 4.0 International License (http://creativecomm ons.org/licenses/by/4.0/), which permits unrestricted use, distribution, and reproduction in any medium, provided you give appropriate credit to the original author(s) and the source, provide a link to the Creative Commons license, and indicate if changes were made.

Funded by $\mathrm{SCOAP}^{3}$.

\section{Appendix A: The amplitude calculations}

The harmonic oscillator wave functions for the ground charmed baryons $B(C)$ in our calculation are

$$
\begin{aligned}
\psi^{B(C)}(0,0,0,0)= & 3^{\frac{3}{4}}\left(\frac{1}{\pi \alpha_{\rho}^{2}}\right)^{\frac{3}{4}}\left(\frac{1}{\pi \alpha_{\lambda}^{2}}\right)^{\frac{3}{4}} \\
& \times \exp \left[-\frac{P_{\rho}^{2}}{2 \alpha_{\rho}^{2}}-\frac{P_{\lambda}^{2}}{2 \alpha_{\lambda}^{2}}\right],
\end{aligned}
$$

where $\mathbf{p}_{\rho}^{B(C)}=\frac{1}{\sqrt{2}}\left(\mathbf{p}_{3(2)}-\mathbf{p}_{5(4)}\right)$ and $\mathbf{p}_{\lambda}^{B(C)}=\frac{1}{\sqrt{6}}\left(\mathbf{p}_{3(2)}+\right.$ $\left.\mathbf{p}_{5(4)}-2 \mathbf{p}_{1(2)}\right)$.

The ground state wave function of the meson is

$\Psi(0,0)=\left(\frac{1}{\pi \beta^{2}}\right)^{\frac{3}{4}} \exp \left[-\frac{\left(\mathbf{p}_{1}-\mathbf{p}_{2}\right)^{2}}{8 \beta^{2}}\right]$

Since all the final states are in the $S$-wave states in this calculations, the momentum space integration $I_{M_{L_{B}} M_{L_{C}}}^{M_{L_{2}}, m, m^{\prime}}(\mathbf{p})$ can be further expressed as $\prod\left(M_{L_{A}}, m, m^{\prime}\right)$.

For the $1 S$ charmonium state decay:

$$
\begin{aligned}
\Pi(0,0,0)= & \left(\frac{1}{\pi \alpha_{\rho}^{2}}\right)^{\frac{3}{2}}\left(\frac{1}{\pi \alpha_{\lambda}^{2}}\right)^{\frac{3}{2}}\left(\frac{1}{\pi \beta^{2}}\right)^{\frac{3}{4}} \\
& \times \exp \left[-\left(\lambda_{4}-\frac{\lambda_{3}^{2}}{4 \lambda_{2}}\right) P_{B}^{2}\right] \\
& \times \frac{\pi^{2}}{16}\left(\frac{1}{\lambda_{1} \lambda_{2}}\right)^{\frac{3}{2}}\left(12 \varpi^{2} P_{B}^{2}-\frac{3}{\lambda_{1}}+\frac{1}{\lambda_{2}}\right), \\
\Pi(0,1,-1)= & \left(\frac{1}{\pi \alpha_{\rho}^{2}}\right)^{\frac{3}{2}}\left(\frac{1}{\pi \alpha_{\lambda}^{2}}\right)^{\frac{3}{2}}\left(\frac{1}{\pi \beta^{2}}\right)^{\frac{3}{4}} \\
& \times \exp \left[-\left(\lambda_{4}-\frac{\lambda_{3}^{2}}{4 \lambda_{2}}\right) P_{B}^{2}\right] \\
& \times \frac{\pi^{2}}{16}\left(\frac{1}{\lambda_{1} \lambda_{2}}\right)^{\frac{3}{2}}\left(\frac{3}{\lambda_{1}}-\frac{1}{\lambda_{2}}\right) \\
= & \Pi(0,-1,1) .
\end{aligned}
$$

For the $1 D$ charmonium state decay:

$$
\begin{aligned}
\Pi(0,0,0)= & \left(\frac{1}{\pi \alpha_{\rho}^{2}}\right)^{\frac{3}{2}}\left(\frac{1}{\pi \alpha_{\lambda}^{2}}\right)^{\frac{3}{2}}\left(\frac{1}{\beta^{2}}\right)^{\frac{7}{4}} \\
& \times \exp \left[-\left(\lambda_{4}-\frac{\lambda_{3}^{2}}{4 \lambda_{2}}\right) P_{B}^{2}\right]
\end{aligned}
$$




$$
\begin{aligned}
& \times \pi^{\frac{5}{4}}\left(\frac{1}{\lambda_{1} \lambda_{2}}\right)^{\frac{3}{2}}\left(\frac{\sqrt{3}}{2} \zeta^{2} \varpi^{2} P_{B}^{4}+\frac{\zeta^{2} P_{B}^{2}}{8 \sqrt{3} \lambda_{2}}\right. \\
& \left.-\frac{\sqrt{3} \zeta^{2} P_{B}^{2}}{8 \lambda_{1}}-\frac{\zeta \varpi P_{B}^{2}}{\sqrt{3} \lambda_{2}}+\frac{\sqrt{3}}{36 \lambda_{2}^{2}}\right), \\
& \Pi(0,1,-1)=\left(\frac{1}{\pi \alpha_{\rho}^{2}}\right)^{\frac{3}{2}}\left(\frac{1}{\pi \alpha_{\lambda}^{2}}\right)^{\frac{3}{2}}\left(\frac{1}{\beta^{2}}\right)^{\frac{7}{4}} \\
& \times \exp \left[-\left(\lambda_{4}-\frac{\lambda_{3}^{2}}{4 \lambda_{2}}\right) P_{B}^{2}\right] \\
& \times \pi^{\frac{5}{4}}\left(\frac{1}{\lambda_{1} \lambda_{2}}\right)^{\frac{3}{2}}\left(\frac{\sqrt{3} \zeta^{2} P_{B}^{2}}{8 \lambda_{1}}-\frac{\zeta^{2} P_{B}^{2}}{8 \sqrt{3} \lambda_{2}}+\frac{\sqrt{3}}{72 \lambda_{2}^{2}}\right) \\
& =\Pi(0,-1,1) \text {, } \\
& \Pi(1,-1,0)=\left(\frac{1}{\pi \alpha_{\rho}^{2}}\right)^{\frac{3}{2}}\left(\frac{1}{\pi \alpha_{\lambda}^{2}}\right)^{\frac{3}{2}}\left(\frac{1}{\beta^{2}}\right)^{\frac{7}{4}} \\
& \times \exp \left[-\left(\lambda_{4}-\frac{\lambda_{3}^{2}}{4 \lambda_{2}}\right) P_{B}^{2}\right] \\
& \times \pi^{\frac{5}{4}}\left(\frac{1}{\lambda_{1} \lambda_{2}}\right)^{\frac{3}{2}}\left(\frac{\zeta \varpi P_{B}^{2}}{4 \lambda_{2}}-\frac{1}{24 \lambda_{2}^{2}}\right) \\
& =\Pi(1,0,-1) \text {, } \\
& \Pi(2,-1,-1)=\left(\frac{1}{\pi \alpha_{\rho}^{2}}\right)^{\frac{3}{2}}\left(\frac{1}{\pi \alpha_{\lambda}^{2}}\right)^{\frac{3}{2}}\left(\frac{1}{\beta^{2}}\right)^{\frac{7}{4}} \\
& \times \exp \left[-\left(\lambda_{4}-\frac{\lambda_{3}^{2}}{4 \lambda_{2}}\right) P_{B}^{2}\right] \\
& \times \pi^{\frac{5}{4}}\left(\frac{1}{\lambda_{1} \lambda_{2}}\right)^{\frac{3}{2}} \frac{\sqrt{2}}{24 \lambda_{2}^{2}} \text {. }
\end{aligned}
$$

Here,

$$
\begin{aligned}
\lambda_{1} & =\frac{1}{\alpha_{\rho}^{2}}, \\
\lambda_{2} & =\frac{1}{\alpha_{\lambda}^{2}}+\frac{1}{3 \beta^{2}}, \\
\lambda_{3} & =-\frac{\sqrt{6}}{9 \beta^{2}}, \\
\lambda_{4} & =\frac{1}{18 \beta^{2}}, \\
\varpi & =\frac{1}{3}-\frac{\lambda_{3}}{2 \sqrt{6} \lambda_{2}}, \\
\zeta & =\frac{1}{3}+\frac{\lambda_{3}}{\sqrt{6} \lambda_{2}},
\end{aligned}
$$

for the above expressions. $\left|P_{B}\right|$ reads as

$$
\left|P_{B}\right|=\frac{\sqrt{\left[m_{A}^{2}-\left(m_{B}+m_{C}\right)^{2}\right]\left[m_{A}^{2}-\left(m_{B}-m_{C}\right)^{2}\right]}}{2 m_{A}} .
$$

With the amplitudes for the $1 S$ states decaying into two $S$-wave final states, we can obtain the radially and orbitally excited states' amplitudes which are related to the lowest radial or orbital states by differentiation [65],

$$
\begin{aligned}
\mathcal{M}_{4 S}= & \frac{1}{3 \sqrt{35}}\left(15 \beta \frac{\partial}{\partial \beta}+6 \beta^{2} \frac{\partial^{2}}{\partial \beta^{2}}+2 \beta^{3} \frac{\partial^{3}}{\partial \beta^{3}}\right) \mathcal{M}_{1 S} \\
\mathcal{M}_{5 S}= & \frac{1}{18 \sqrt{70}}\left(63+72 \beta \frac{\partial}{\partial \beta}+96 \beta^{2} \frac{\partial^{2}}{\partial \beta^{2}}+24 \beta^{3} \frac{\partial^{3}}{\partial \beta^{3}}\right. \\
& \left.+4 \beta^{4} \frac{\partial^{4}}{\partial \beta^{4}}\right) \mathcal{M}_{1 S} \\
\mathcal{M}_{6 S}= & \frac{1}{45 \sqrt{77}}\left(\frac{675}{2} \beta \frac{\partial}{\partial \beta}+240 \beta^{2} \frac{\partial^{2}}{\partial \beta^{2}}+120 \beta^{3} \frac{\partial^{3}}{\partial \beta^{3}}\right. \\
& \left.+20 \beta^{4} \frac{\partial^{4}}{\partial \beta^{4}}+2 \beta^{5} \frac{\partial^{5}}{\partial \beta^{5}}\right) \mathcal{M}_{1 S} \\
\mathcal{M}_{3 D}= & \frac{1}{3 \sqrt{14}}\left(7+2 \beta \frac{\partial}{\partial \beta}+2 \beta^{2} \frac{\partial^{2}}{\partial \beta^{2}}\right) \mathcal{M}_{1 D} \\
\mathcal{M}_{4 D}= & \frac{1}{3 \sqrt{231}}\left(27 \beta \frac{\partial}{\partial \beta}+6 \beta^{2} \frac{\partial^{2}}{\partial \beta^{2}}+2 \beta^{3} \frac{\partial^{3}}{\partial \beta^{3}}\right) \mathcal{M}_{1 D} \\
\mathcal{M}_{5 D}= & \frac{1}{6 \sqrt{6006}}\left(231+120 \beta \frac{\partial}{\partial \beta}+144 \beta^{2} \frac{\partial^{2}}{\partial \beta^{2}}+24 \beta^{3} \frac{\partial^{3}}{\partial \beta^{3}}\right. \\
& \left.+4 \beta^{4} \frac{\partial^{4}}{\partial \beta^{4}}\right) \mathcal{M} 1 D
\end{aligned}
$$

\section{References}

1. S.K. Choi et al. [Belle Collaboration], Observation of a narrow charmonium-like state in exclusive $B^{+-} \rightarrow K^{+-} \pi^{+} \pi^{-} J / \Psi$ decays. Phys. Rev. Lett. 91, 262001 (2003)

2. C. Patrignani et al. [Particle Data Group], Review of particle physics. Chin. Phys. C 40, 100001 (2016)

3. H.X. Chen, W. Chen, X. Liu, S.L. Zhu, The hidden-charm pentaquark and tetraquark states. Phys. Rep. 639, 1 (2016)

4. X.L. Wang et al. [Belle Collaboration], Observation of two resonant structures in $e^{+} e^{-} \rightarrow \pi^{+} \pi^{-} \psi(2 S)$ via initial state radiation at Belle. Phys. Rev. Lett. 99, 142002 (2007)

5. J.P. Lees et al. [BaBar Collaboration], Study of the reaction $e^{+} e^{-} \rightarrow \psi(2 S) \pi^{-} \pi^{-}$via initial-state radiation at BaBar. Phys. Rev. D 89, 111103 (2014)

6. G. Pakhlova et al. [Belle Collaboration], Observation of a nearthreshold enhancement in the $e^{+} e^{-} \rightarrow \Lambda_{c}^{+} \Lambda_{c}^{-}$cross section using initial-state radiation. Phys. Rev. Lett. 101, 172001 (2008)

7. D.V. Bugg, An alternative fit to Belle mass spectra for $D \bar{D}, D^{*} \bar{D}^{*}$ and $\Lambda_{c} \bar{\Lambda}_{c}$. J. Phys. G 36, 075002 (2009)

8. G. Cotugno, R. Faccini, A.D. Polosa, C. Sabelli, Charmed baryonium. Phys. Rev. Lett. 104, 132005 (2010)

9. F.K. Guo, J. Haidenbauer, C. Hanhart, U.G. Meissner, Reconciling the $X$ (4630) with the $Y(4660)$. Phys. Rev. D 82, 094008 (2010)

10. B.Q. Li, K.T. Chao, Higher charmonia and $X, Y, Z$ states with screened potential. Phys. Rev. D 79, 094004 (2009)

11. G.J. Ding, J.J. Zhu, M.L. Yan, Canonical charmonium interpretation for Y(4360) and Y(4660). Phys. Rev. D 77, 014033 (2008)

12. L.C. Gui, L.S. Lu, Q.F. Lü, X.H. Zhong, Q. Zhao, Strong decays of higher charmonium states into open-charm meson pairs. arXiv:1801.08791 [hep-ph]

13. M.N. Anwar, Y. Lu, B.S. Zou, Modeling charmonium- $\eta$ decays of $J^{P C}=1^{-}$higher charmonia. Phys. Rev. D 95, 114031 (2017)

14. L. Maiani, F. Piccinini, A.D. Polosa, V. Riquer, The Z(4430) and a new paradigm for spin interactions in tetraquarks. Phys. Rev. D 89, 114010 (2014)

15. D. Ebert, R.N. Faustov, V.O. Galkin, Excited heavy tetraquarks with hidden charm. Eur. Phys. J. C 58, 399 (2008) 
16. Z.G. Wang, Vector tetraquark state candidates: $Y(4260 / 4220)$, $Y(4360 / 4320), Y(4390)$ and $Y(4660 / 4630)$. arXiv:1803.05749 [hep-ph]

17. Q.F. Lü, Y.B. Dong, $X(4140), X(4274), X(4500)$, and $X(4700)$ in the relativized quark model. Phys. Rev. D 94, 074007 (2016)

18. W. Chen, S.L. Zhu, The vector and axial-vector charmonium-like states. Phys. Rev. D 83, 034010 (2011)

19. J.R. Zhang, M.Q. Huang, The $P$-wave $[c s][\bar{c} \bar{s}]$ tetraquark state: $Y(4260)$ or $Y(4660)$ ? Phys. Rev. D 83, 036005 (2011)

20. R.M. Albuquerque, M. Nielsen, QCD sum rules study of the $J^{P C}=$ $1^{-}$charmonium Y mesons. Nucl. Phys. A 815, 53 (2009). (Erratum: [Nucl. Phys. A 857, 48 (2011)])

21. Z.G. Wang, Analysis of the $Z_{c}(4020), Z_{c}(4025), Y(4360)$ and $Y(4660)$ as vector tetraquark states with QCD sum rules. Eur. Phys. J. C 74, 2874 (2014)

22. Z.G. Wang, Tetraquark state candidates: $Y(4260), Y(4360)$, $Y(4660)$ and $Z_{c}(4020 / 4025)$. Eur. Phys. J. C 76, 387 (2016)

23. H. Sundu, S.S. Agaev, K. Azizi, The resonance $Y(4660)$ as a vector tetraquark and its strong decay channels. arXiv:1805.04705 [hep$\mathrm{ph}]$

24. F.K. Guo, C. Hanhart, U.G. Meissner, Evidence that the Y(4660) is a $f_{0}(980) \psi^{\prime}$ bound state. Phys. Lett. B 665, 26 (2008)

25. F.K. Guo, C. Hanhart, U.G. Meissner, Implications of heavy quark spin symmetry on heavy meson hadronic molecules. Phys. Rev. Lett. 102, 242004 (2009)

26. Z.G. Wang, X.H. Zhang, Analysis of $Y(4660)$ and related bound states with QCD sum rules. Commun. Theor. Phys. 54, 323 (2010)

27. R.M. Albuquerque, M. Nielsen, R. Rodrigues da Silva, Exotic $1^{-}$ states in QCD sum rules. Phys. Rev. D 84, 116004 (2011)

28. C.F. Qiao, A uniform description of the states recently observed at B-factories. J. Phys. G 35, 075008 (2008)

29. S. Dubynskiy, M.B. Voloshin, Hadro-charmonium. Phys. Lett. B 666, 344 (2008)

30. E. van Beveren, G. Rupp, Evidence for further charmonium vector resonances. Chin. Phys. C 35, 319 (2011)

31. B. Aubert et al. [BaBar Collaboration], Exclusive initial-stateradiation production of the $D \bar{D}, D^{*} \bar{D}$, and $D^{*} \bar{D}^{*}$ systems. Phys. Rev. D 79, 092001 (2009)

32. X. Liu, H.W. Ke, X. Liu, X.Q. Li, Study of structures and dynamical decay mechanisms for multiquark systems. Phys. Rev. D 93, 074013 (2016)

33. X. Liu, H.W. Ke, X. Liu, X.Q. Li, Exploring open-charm decay mode $\Lambda_{c} \bar{\Lambda}_{c}$ of charmonium-like state $Y(4630)$. Eur. Phys. J. C 76, $549(2016)$

34. X.D. Guo, D.Y. Chen, H.W. Ke, X. Liu, X.Q. Li, Study on the rare decays of $Y(4630)$ induced by final state interactions. Phys. Rev. D 93, 054009 (2016)

35. Y.Y. Wang, Q.F. Lü, E. Wang, D.M. li, Role of $Y(4630)$ in the $p \bar{p} \rightarrow \Lambda_{c} \bar{\Lambda}_{c}$ reaction near threshold. Phys. Rev. D 94, 014025 (2016)

36. N. Lee, Z.G. Luo, X.L. Chen, S.L. Zhu, Possible deuteron-like molecular states composed of heavy baryons. Phys. Rev. D 84, 014031 (2011)

37. Y.A. Simonov, Theory of hadron decay into baryon-antibaryon final state. Phys. Rev. D 85, 105025 (2012)

38. E. van Beveren, X. Liu, R. Coimbra, G. Rupp, Possible $\psi(5 S)$, $\psi(4 D), \psi(6 S)$ and $\psi(5 D)$ signals in $\Lambda_{c} \bar{\Lambda}_{c}$. EPL 85, $61002(2009)$

39. L. Micu, Decay rates of meson resonances in a quark model. Nucl. Phys. B 10, 521 (1969)

40. R.D. Carlitz, M. Kislinger, Regge amplitude arising from su(6)w vertices. Phys. Rev. D 2, 336 (1970)
41. A. Le Yaouanc, L. Oliver, O. Pene, J.C. Raynal, Naive quark pair creation model of strong interaction vertices. Phys. Rev. D 8, 2223 (1973)

42. A. Le Yaouanc, L. Oliver, O. Pene, J.C. Raynal, Hadron Transitions in the Quark Model (Gordon and Breach, New York, 1988)

43. A. Le Yaouanc, L. Oliver, O. Pene, J.-C. Raynal, Strong decays of $\psi(4.028)$ as a radial excitation of charmonium. Phys. Lett. 71B, 397 (1977)

44. A. Le Yaouanc, L. Oliver, O. Pene, J.-C. Raynal, Why is $\psi(4.414)$ so narrow? Phys. Lett. 72B, 57 (1977)

45. P. Geiger, E.S. Swanson, Distinguishing among strong decay models. Phys. Rev. D 50, 6855 (1994)

46. E.S. Ackleh, T. Barnes, E.S. Swanson, On the mechanism of open flavor strong decays. Phys. Rev. D 54, 6811 (1996)

47. F.E. Close, E.S. Swanson, Dynamics and decay of heavy-light hadrons. Phys. Rev. D 72, 094004 (2005)

48. E. Eichten, K. Gottfried, T. Kinoshita, K.D. Lane, T.M. Yan, Charmonium: comparison with experiment. Phys. Rev. D 21, 203 (1980)

49. S. Godfrey, N. Isgur, Mesons in a relativized quark model with chromodynamics. Phys. Rev. D 32, 189 (1985)

50. J. Segovia, D.R. Entem, F. Fernandez, Charm spectroscopy beyond the constituent quark model. arXiv:0810.2875 [hep-ph]

51. A.M. Badalian, B.L.G. Bakker, I.V. Danilkin, The $S-D$ mixing and di-electron widths of higher charmonium $1^{-}$states. Phys. Atom. Nucl. 72, 638 (2009)

52. T. Barnes, S. Godfrey, E.S. Swanson, Higher charmonia. Phys. Rev. D 72, $054026(2005)$

53. W.J. Deng, H. Liu, L.C. Gui, X.H. Zhong, Charmonium spectrum and their electromagnetic transitions with higher multipole contributions. Phys. Rev. D 95, 034026 (2017)

54. C. Hayne, N. Isgur, Beyond the wave function at the origin: some momentum dependent effects in the nonrelativistic quark model. Phys. Rev. D 25, 1944 (1982)

55. S.C. Xue, G.Y. Wang, E. Wang, D.M. Li, The possible members of the $5^{1} S_{0}$ meson nonet. Eur. Phys. J. C 78, 479 (2018)

56. R. Kokoski, N. Isgur, Meson decays by flux tube breaking. Phys. Rev. D 35, 907 (1987)

57. H.G. Blundell, S. Godfrey, The $\Xi(2220)$ revisited: strong decays of the $1^{3} F_{2} 1^{3} F_{4} s \bar{s}$ mesons. Phys. Rev. D 53, 3700 (1996)

58. K.L. Wang, L.Y. Xiao, X.H. Zhong, Q. Zhao, Understanding the newly observed $\Omega_{c}$ states through their decays. Phys. Rev. D 95, $116010(2017)$

59. L.Y. Xiao, K.L. Wang, Q.F. Lü, X.H. Zhong, S.L. Zhu, Strong and radiative decays of the doubly charmed baryons. Phys. Rev. D 96, 094005 (2017)

60. L.Y. Xiao, Q.F. Lü, S.L. Zhu, Strong decays of the $1 P$ and $2 D$ doubly charmed states. Phys. Rev. D 97, 074005 (2018)

61. J. Segovia, D.R. Entem, F. Fernandez, Scaling of the ${ }^{3} P_{0}$ strength in heavy meson strong decays. Phys. Lett. B 715, 322 (2012)

62. S. Godfrey, K. Moats, Bottomonium mesons and strategies for their observation. Phys. Rev. D 92, 054034 (2015)

63. H.G. Blundell, Meson properties in the quark model: a look at some outstanding problems. arXiv:hep-ph/9608473

64. D.M. Li, P.F. Ji, B. Ma, The newly observed open-charm states in quark model. Eur. Phys. J. C 71, 1582 (2011)

65. J.F. Liu, G.J. Ding, Bottomonium spectrum with coupled-channel effects. Eur. Phys. J. C 72, 1981 (2012) 